Kragujevac Journal of Mathematics

Volume 44(3) (2020), Pages 431-442.

\title{
ON THE NORMALIZED LAPLACIAN SPECTRUM OF SOME GRAPHS
}

\author{
RENNY P. VARGHESE ${ }^{1}$ AND D. SUSHA ${ }^{1}$
}

\begin{abstract}
In this paper we determine the normalized Laplacian spectrum of duplication vertex join of two graphs, duplication graph, splitting graph and double graph of a regular graph. Here we investigate some graph invariants like the normalized Laplacian energy, Kemeny's constant and number of spanning tree of these graphs.
\end{abstract}

\section{INTRODUCTION}

All graphs explained in this paper are undirected, without parallel edges and loops. Let $G=G(V, E)$ be a graph with vertex set $V(G)=\left\{v_{1}, v_{2}, \ldots, v_{n}\right\}$ and edge set $E(G)$. The adjacency matrix, $A(G)=\left(a_{i j}\right)_{n \times n}$, is an $n \times n$ symmetric matrix with rows and columns are indexed by vertices of $G$ where $a_{i j}=1$ if the vertices $v_{i}$ and $v_{j}$ are adjacent in $G, 0$ elsewhere. The characteristic polynomial of $A$ is of the form $f_{G}(A: x)=\operatorname{det}\left(x I_{n}-A\right)$ where $I_{n}$ is the identity matrix of order $n$. The roots of $f_{G}(A: x)=0$ constitute the eigenvalues of $G$. We denote these as $\lambda_{1} \geq \lambda_{2} \geq \cdots \geq \lambda_{n}$ and form the $A$ - spectrum of $G$.

Let $d_{i}$ be the degree of the vertex $v_{i}$ in $G$ and $D(G)=\operatorname{diag}\left(d_{1}, d_{2}, \ldots, d_{n}\right)$ be the diagonal degree matrix of $G$. The matrix $D^{-1 / 2}$ is a diagonal matrix with diagonal entries $\frac{1}{\sqrt{d_{i}}}$ for all $i$. Chung in [5] introduced a new matrix called, normalized Laplacian matrix of a graph $G$. It is defined to be the matrix $\tilde{L}(G)=D^{-1 / 2} L D^{-1 / 2}$, whose

Key words and phrases. Normalized Laplacian spectrum, normalized Laplacian energy, Kemeny's constant, spanning tree.

2010 Mathematics Subject Classification. Primary: 05C50, 05C90.

DOI 10.46793/KgJMat2003.431V

Received: December 21, 2017.

Accepted: June 04, 2018. 
$(i, j)^{t h}$ - entry is given by,

$$
\tilde{L}_{i j}= \begin{cases}1, & \text { if } v_{i}=v_{j} \text { and } d_{i} \neq 0 \\ \frac{-1}{\sqrt{d_{i} d_{j}}}, & \text { if } v_{i} \text { and } v_{j} \text { are adjacent } \\ 0 & \text { otherwise }\end{cases}
$$

The roots of the characteristic equation of $\tilde{L}$ are known as the normalized Laplacian eigenvalues of $G$. Since $\tilde{L}(G)$ is symmetric and positive semi definite matrix, its eigenvalues are all real and non negative of the form $0=\sigma_{1} \leq \sigma_{2} \leq \cdots \leq \sigma_{n}$. These eigenvalues together their multiplicities is called normalized Laplacian spectrum or $\tilde{L}$-spectrum of $G$ and is denoted by $\tilde{\operatorname{LSpec}}(G)$.

The mathematicians like Chen and Zhang express the resistance distance in terms of normalized Laplacian eigenvalues and vectors of the graph $G$ [4]. Also they propose degree-Kirchhoff index is closely related to spectrum of the normalized Laplacian. The concept of limit point for the normalized Laplacian eigenvalues are used by Kirkkland in [9]. In [1] Banergee and Jost investigated, how the normalized spectrum is affected by some operations like mofit doubling, graph splitting or joining. Renny and Susha defined some new join and corona based on duplication graph of an arbitrary graph $($ see $[13,14])$.

Motivated by these, in this paper we are interested in finding the normalized Laplacian spectrum of duplication, splitting and double graph of a regular graph $G$. Also we define and determine the normalized Laplacian spectrum of Duplication vertex join of two regular graphs $G_{1}$ and $G_{2}$.

The arrangement of the paper in section wise as follows. Section 2 describes the necessary preliminaries. In Section 3, we determine the normalized Laplacian spectrum of duplication vertex join of two graphs, duplication, splitting, double graph of a regular graph. Then in the last section we discuss some applications such as normalized Laplacian energy, the Kemeny's constant and number of spanning tree of these graphs.

\section{Preliminaries}

Definition 2.1 ([8,11,12]). Let $G$ be a graph with vertex set $V(G)=\left\{v_{1}, v_{2}, \ldots, v_{n}\right\}$ and $U(G)=\left\{u_{1}, u_{2}, \ldots, u_{n}\right\}$ be the vertex set of another copy of $G$. The double graph, $D_{2}(G)$, is the graph obtained by joining $u_{i}$ to every vertices in $\mathcal{N}\left(v_{i}\right)$, the neighbourhood set of $v_{i}$ of $G$, for each $i$. If we remove the edges of the copy of $G$ in vertex set $U(G)$ in the double graph we get the splitting graph, $\operatorname{splt}(G)$, of $G$. Removing the edges of two copies of $G$ in the double graph, then it is called the duplication graph, $\mathcal{D G}$, of $G$.

Lemma 2.1 ([6]). Let $M=\left[\begin{array}{ll}M_{1} & M_{2} \\ M_{2} & M_{1}\end{array}\right]$ be a symmetric block matrix of order $2 \times 2$. Then the eigenvalues of $M$ are those of $M_{1}+M_{2}$ together with $M_{1}-M_{2}$. 
Proposition $2.1([6])$. Let $P_{0}, P_{1}, P_{2}$ and $P_{3}$ be matrices of order $n_{1} \times n_{1}, n_{1} \times n_{2}, n_{2} \times$ $n_{1}, n_{2} \times n_{2}$ respectively. Then

$$
\operatorname{det}\left[\begin{array}{ll}
P_{0} & P_{1} \\
P_{2} & P_{3}
\end{array}\right]=\left\{\begin{array}{l}
\operatorname{det}\left(P_{0}\right) \operatorname{det}\left(P_{3}-P_{2} P_{0}^{-1} P_{1}\right), \quad \text { if } P_{0} \text { is invertible } \\
\operatorname{det}\left(P_{3}\right) \operatorname{det}\left(P_{0}-P_{1} P_{3}^{-1} P_{2}\right), \quad \text { if } P_{3} \text { is invertible. }
\end{array}\right.
$$

Remark 2.1. Let $G$ be a $r$-regular graph with adjacency matrix $A$. Then normalized Laplacian matrix is $I-\frac{A}{r}[5]$.
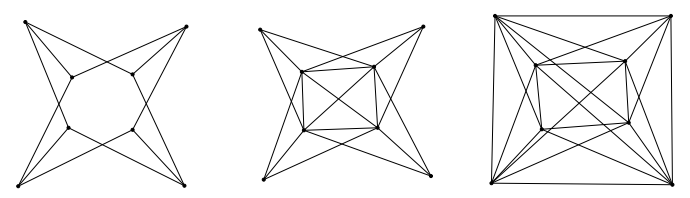

FiguRE 1. Duplication, splitting and double graph of $K_{4}$

\section{Normalized Laplacian Spectrum of Some Graphs}

In this section we determine the normalized Laplacian spectrum of duplication vertex join of two graphs, duplication, double and splitting graph of a regular graph.

\subsection{Normalized Laplacian spectrum of duplication vertex join.}

Definition 3.1. For $i=1,2$, let $G_{i}$ be graphs on $n_{i}$ vertices. Let $\mathcal{D} \mathcal{G}_{1}$ be the duplication graph of $G_{1}$. The duplication vertex join of $G_{1}$ and $G_{2}$ is denoted by $G_{1} \nabla G_{2}$ and is the graph obtained from $\mathcal{D} \mathcal{G}_{1}$ and $G_{2}$, by joining every vertex of $G_{1}$ to all the vertices of $G_{2}$.

Example 3.1. The following, Figure 2 illustrate the Definition 3.1.

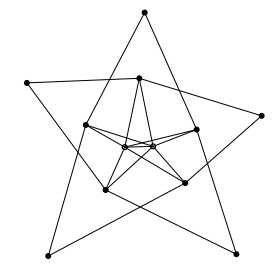

Figure 2. Duplication vertex join of $C_{5}$ and $K_{2}$.

Let $G_{i}, i=1,2$, be $r_{i}$-regular graphs on $n_{i}$ vertices and $m_{i}$ edges. Then $G_{1} \bar{\nabla} G_{2}$ has $2 n_{1}+n_{2}$ vertices and $2 m_{1}+m_{2}+n_{1} n_{2}$ edges. 
Theorem 3.1. For $i=1,2$, let $G_{i}$ be $r_{i}$-regular graphs on $n_{i}$ vertices with spectrum $\lambda_{i 1}(G) \geq \lambda_{i 2}(G) \geq \cdots \geq \lambda_{i n_{i}}(G)$. Then the normalized Laplacian spectrum of $G_{1} \bar{\nabla} G_{2}$ is $0,1-\frac{\lambda_{2 k}}{n_{1}+r_{2}}, 1 \pm \frac{\lambda_{1 i}}{\sqrt{r_{1}\left(n_{2}+r_{1}\right)}}, i=2,3, \ldots, n_{1}, k=2,3, \ldots, n_{2}$. Together with the roots of the equation

$$
x^{2}-\frac{3 n_{1}+2 r_{2}}{n_{1}+r_{2}} x+\frac{2 n_{1} n_{2}+2 n_{1} r_{1}+n_{2} r_{2}}{\left(n_{1}+r_{2}\right)\left(n_{2}+r_{1}\right)}=0 .
$$

Proof. Let $G_{i}, i=1,2$, be $r_{i}$-regular graphs on $n_{i}$ vertices. Let $V\left(G_{1}\right)=$ $\left\{v_{1}, v_{2}, \ldots, v_{n_{1}}\right\}$ be the vertex set of $G_{1}$ and $U\left(G_{1}\right)=\left\{x_{1}, x_{2}, \ldots, x_{n_{1}}\right\}$ is the additional vertices corresponding to each vertex of $G_{1}$. Let $V\left(G_{2}\right)=\left\{u_{1}, u_{2}, \ldots, u_{n_{2}}\right\}$ be the vertex set of $G_{2}$.

Under this vertex partitioning the adjacency matrix of $G_{1} \bar{\nabla} G_{2}$ is,

$$
A=\left[\begin{array}{ccc}
0_{n_{1}} & A_{1} & J_{n_{1} \times n_{2}} \\
A_{1} & 0_{n_{1}} & 0_{n_{1} \times n_{2}} \\
J_{n_{2} \times n_{1}} & 0_{n_{2} \times n_{1}} & A_{2}
\end{array}\right],
$$

where $A_{1}$ and $A_{2}$ are the adjacency matrix of $G_{1}$ and $G_{2}$ respectively. $J$ denote matrix with all entries equal to 1 and 0 is the zero matrix of appropriate order. The degree of the vertices of $G_{1} \bar{\nabla} G_{2}$ are $d_{G_{1} \bar{\nabla} G_{2}}\left(v_{i}\right)=n_{2}+r_{1}, d_{G_{1} \bar{\nabla} G_{2}}\left(x_{i}\right)=r_{1}, i=1,2, \ldots, n_{1}$ and $d_{G_{1} \bar{\nabla} G_{2}}\left(u_{j}\right)=n_{1}+r_{2}, j=1,2, \ldots, n_{2}$.

The diagonal degree matrix of $G_{1} \bar{\nabla} G_{2}$ is

$$
D=\left[\begin{array}{ccc}
\left(r_{1}+n_{2}\right) I_{n_{1}} & 0 & 0 \\
0 & r_{1} I_{n_{1}} & 0 \\
0 & 0 & \left(n_{1}+r_{2}\right) I_{n_{2}}
\end{array}\right]
$$

Hence, the Laplace adjacency matrix of $G_{1} \bar{\nabla} G_{2}$ is

$$
L=\left[\begin{array}{ccc}
\left(r_{1}+n_{2}\right) I & -A_{1} & -J_{n_{1} \times n_{2}} \\
-A_{1} & r_{1} I & 0_{n_{1} \times n_{2}} \\
-J_{n_{2} \times n_{1}} & 0_{n_{2} \times n_{1}} & n_{1} I_{n_{2}}+L_{2}
\end{array}\right]
$$

where $L_{2}$ is the Laplacian matrix of $G_{2}$. Also,

$$
D^{-1 / 2}=\left[\begin{array}{ccc}
\frac{I_{n_{1}}}{\sqrt{r_{1}+n_{2}}} & 0 & 0 \\
0 & \frac{I_{n_{1}}}{\sqrt{r_{1}}} & 0 \\
0 & 0 & \frac{I_{n_{2}}}{\sqrt{n_{1}+r_{2}}}
\end{array}\right] \text {. }
$$


By simple calculation we get

$$
D^{-1 / 2} L D^{-1 / 2}=\tilde{L}=\left[\begin{array}{ccc}
I_{n_{1}} & \frac{-A_{1}}{\sqrt{r_{1}\left(n_{2}+r_{1}\right)}} & \frac{-J_{n_{1} \times n_{2}}}{\sqrt{\left(n_{1}+r_{2}\right)\left(n_{2}+r_{1}\right)}} \\
\frac{-A_{1}}{\sqrt{r_{1}\left(n_{2}+r_{1}\right)}} & I_{n_{1}} & 0 \\
\frac{-J_{n_{2} \times n_{1}}}{\sqrt{\left(n_{1}+r_{2}\right)\left(n_{2}+r_{1}\right)}} & 0 & I_{n_{2}}-\frac{A_{2}}{n_{1}+r_{2}}
\end{array}\right] .
$$

Since $G_{i}$ is $r_{i}$-regular, it has an eigenvector $\mathbf{j}_{n_{i}}$, a vector with all entries equal to 1 , corresponding to the eigenvalue $r_{i}$. All other eigenvectors are orthogonal to $\mathbf{j}_{n_{i}}$. Let $\lambda_{2 i}$ be an eigenvalue of $G_{2}$ with eigenvector $Z$ such that $\mathbf{j}_{n_{2}}^{T} Z=0$ Then $(0,0, Z)^{T}$ is an eigenvector of $\tilde{L}$ corresponding to the eigenvalue $1-\frac{\lambda_{2 i}}{n_{1}+r_{2}}$.

This is because,

$$
\tilde{L}\left(\begin{array}{l}
0 \\
0 \\
Z
\end{array}\right)=\left(\begin{array}{c}
0 \\
0 \\
Z-\frac{A_{2} Z}{n_{1}+r_{2}}
\end{array}\right)=\left(1-\frac{\lambda_{2 i}}{n_{1}+r_{2}}\right)\left(\begin{array}{l}
0 \\
0 \\
Z
\end{array}\right)
$$

Therefore, $1-\frac{\lambda_{2 i}}{n_{1}+r_{2}}$ for $i=2,3, \ldots, n_{2}$, is an eigenvalue corresponding to the eigenvector $(0,0, Z)^{T}$.

Let $X$ be an eigenvector corresponding to the eigenvalue $\lambda_{1 i}$ of $G_{1}$. Then $(X, X, 0)^{T}$ is an eigenvector corresponding to the eigenvalue $1-\frac{\lambda_{1 i}}{\sqrt{r_{1}\left(n_{2}+r_{1}\right)}}$. For,

$$
\tilde{L}\left(\begin{array}{c}
X \\
X \\
0
\end{array}\right)=\left(\begin{array}{c}
X-\frac{A_{1} X}{\sqrt{\left(r_{1}\left(n_{2}+r_{1}\right)\right.}} \\
\frac{-A_{1} X}{\sqrt{\left(r_{1}\left(n_{2}+r_{1}\right)\right.}}+X \\
0
\end{array}\right)=\left(1-\frac{\lambda_{1 i}}{\sqrt{r_{1}\left(n_{2}+r_{1}\right)}}\right)\left(\begin{array}{c}
X \\
X \\
0
\end{array}\right) .
$$

Therefore, $1-\frac{\lambda_{1 i}}{\sqrt{r_{1}\left(n_{2}+r_{1}\right)}}$ for $i=2,3, \ldots, n_{1}$, is an eigenvalue corresponding to the eigenvector $(X, X, 0)^{T}$. Similarly we can prove $(-X, X, 0)^{T}$ is an eigenvector corresponding to the eigenvalue $1+\frac{\lambda_{1 i}}{\sqrt{r_{1}\left(n_{2}+r_{1}\right)}}$ for $i=2,3, \ldots, n_{1}$.

Thus we obtain $n_{2}-1+2\left(n_{1}-1\right)=2 n_{1}+n_{2}-3$ eigenvalues of $\tilde{L}$ all orthogonal to $(\mathbf{j}, 0,0)^{T},(0, \mathbf{j}, 0)^{T}$ and $(0,0, \mathbf{j})^{T}$. 
The remaining three vectors of $\tilde{L}$ are of the form $\tau=(\alpha \mathbf{j}, \beta \mathbf{j}, \gamma \mathbf{j})^{T}$ for $(\alpha, \beta, \gamma) \neq$ $(0,0,0)$. Let $v$ be an eigenvalue of $\tilde{L}$ with eigenvector $\tau$. Then from $\tilde{L} \tau=v \tau$ we get,

$$
\begin{array}{r}
\alpha-\frac{r_{1}}{\sqrt{r_{1}\left(n_{2}+r_{1}\right)}} \beta-\frac{n_{2}}{\sqrt{\left(n_{1}+r_{2}\right)\left(n_{2}+r_{1}\right)}} \gamma=v \alpha, \\
-\frac{r_{1}}{\sqrt{r_{1}\left(n_{2}+r_{1}\right)}} \alpha+\beta+0 \gamma=v \beta, \\
-\frac{n_{1}}{\sqrt{\left(n_{1}+r_{2}\right)\left(n_{2}+r_{1}\right)}} \alpha+0 \beta+\left(1-\frac{r_{2}}{n_{1}+r_{2}} \gamma=v \gamma .\right.
\end{array}
$$

By solving above three equations we get the cubic equation as,

$$
x^{3}-\frac{3 n_{1}+2 r_{2}}{n_{1}+r_{2}} x^{2}+\frac{2 n_{1} n_{2}+2 n_{1} r_{1}+n_{2} r_{2}}{\left(n_{1}+r_{2}\right)\left(n_{2}+r_{1}\right)} x=0 .
$$

Now the theorem follows.

Corollary 3.1. If $G_{2} \cong \bar{K}_{n_{2}}$ (totally disconnected graph with $n_{2}$ vertices), then the normalized Laplacian of $G_{1} \nabla G_{2}$ consists of $0,2, \alpha_{i}$ and $\beta_{i}$ together with 1 , repeats $n_{2}$ times, where $\alpha_{i}=1-\frac{\lambda_{1 i}}{\sqrt{r_{1}\left(n_{2}+r_{1}\right)}}, \beta_{i}=1+\frac{\lambda_{1 i}}{\sqrt{r_{1}\left(n_{2}+r_{1}\right)}}, i=2,3, \ldots, n_{1}$.

Proof. If $G_{2}$ is totally disconnected or $\bar{K}_{n_{2}}$ then $r_{2}=0$. The cubic equation (3.4) reduces to

$$
x^{3}-3 x^{2}+2 x=0 .
$$

On solving we get the solution as $x=0,1,2$. The remaining eigenvalues are obtained from Theorem 3.1. Hence the corollary is proved.

\subsection{Normalized Laplacian spectrum of duplication, splitting and double graph.}

Theorem 3.2. Let $G$ be a $r$-regular graph on $n$ vertices with adjacency spectrum $\left\{r=\lambda_{1}, \lambda_{2}, \ldots, \lambda_{n}\right\}$. Then the normalized Laplacian spectrum of the duplication graph, $\mathcal{D G}$, consists of $1 \pm \frac{\lambda_{i}}{r}$ for $i=1,2, \ldots, n$.

Proof. Let $A$ be the adjacency matrix of $G$. The Laplacian and normalized Laplacian matrix of $\mathcal{D} \mathcal{G}$ are

$$
L=\left[\begin{array}{cc}
r I_{n} & -A \\
-A & r I_{n}
\end{array}\right] \text { and } \tilde{L}=\left[\begin{array}{cc}
I_{n} & \frac{-A}{r} \\
\frac{-A}{r} & I_{n}
\end{array}\right]
$$

Since $G$ is $r$-regular with $n$ vertices, the duplication graph $\mathcal{D G}$ is also an $r$-regular graph on $2 n$ vertices with eigenvalues $\pm \lambda_{i}, i=1,2, \ldots, n$. By Remark 2.1, the normalized Laplacian eigenvalues of $\mathcal{D G}$ are $1 \pm \frac{\lambda_{i}}{r}, i=1,2, \ldots, n$.

Theorem 3.3. Let $G$ be an $r$-regular graph on $n$ vertices with adjacency spectrum $\left\{r=\lambda_{1}, \lambda_{2}, \ldots, \lambda_{n}\right\}$. Then the normalized Laplacian spectrum of the splitting graph, $\operatorname{splt}(G)$, consists of $1-\frac{\lambda_{i}}{r}, 1+\frac{\lambda_{i}}{2 r}$ for $i=1,2, \ldots, n$. 
Proof. Let $A$ and $D$ be respectively the adjacency matrix and diagonal degree matrix of $G$. The Laplacian matrix of $\operatorname{splt}(G)$ is $L=\left[\begin{array}{cc}2 r I_{n}-A & -A \\ -A & r I_{n}\end{array}\right]$.

Also $D=\left[\begin{array}{cc}2 r I_{n} & 0 \\ 0 & r I_{n}\end{array}\right]$ and $D^{-1 / 2}=\left[\begin{array}{cc}\frac{I_{n}}{\sqrt{2 r}} & 0 \\ 0 & \frac{I_{n}}{\sqrt{r}}\end{array}\right]$.

The normalized Laplacian matrix is

$$
\tilde{L}=D^{-1 / 2} L D^{-1 / 2}=\left[\begin{array}{cc}
I_{n}-\frac{A}{2 r} & \frac{-A}{r \sqrt{2}} \\
\frac{-A}{r \sqrt{2}} & I_{n}
\end{array}\right] .
$$

The characteristic polynomial of $\tilde{L}$ is

$$
\operatorname{det}(x I-\tilde{L})=\operatorname{det}\left[\begin{array}{cc}
(x-1) I_{n}+\frac{A}{2 r} & \frac{A}{r \sqrt{2}} \\
\frac{A}{r \sqrt{2}} & (x-1) I_{n}
\end{array}\right] .
$$

Using Proposition 2.1 and the result [6] that, if $\lambda_{i}$ is an eigenvalue of $A$ then $P\left(\lambda_{i}\right)$ is an eigenvalue of $P(A)$, for any polynomial $P(x)$. We arrive at

$$
\begin{aligned}
f_{G}(\tilde{L}: x) & =(x-1)^{n} \operatorname{det}\left((x-1) I_{n}+\frac{A}{2 r}-\frac{A^{2}}{2 r^{2}(x-1)}\right) \\
& =\operatorname{det}\left((x-1)^{2} I_{n}+(x-1) \frac{A}{2 r}-\frac{A^{2}}{2 r^{2}}\right) \\
& =\prod_{i=1}^{n}\left((x-1)^{2}+(x-1) \frac{\lambda_{i}}{2 r}-\frac{\lambda_{i}^{2}}{2 r^{2}}\right) \\
& =\prod_{i=1}^{n}\left(x^{2}-\left(\frac{4 r-\lambda_{i}}{2 r}\right) x+\frac{2 r^{2}-r \lambda_{i}-\lambda_{i}^{2}}{2 r^{2}}\right) \\
& =\prod_{i=1}^{n}\left(x-1-\frac{\lambda_{i}}{2 r}\right)\left(x-1+\frac{\lambda_{i}}{r}\right) .
\end{aligned}
$$

Thus we obtain the normalized Laplacian spectrum.

Theorem 3.4. Let $G$ be an $r$-regular graph on $n$ vertices with adjacency spectrum $\left\{r=\lambda_{1}, \lambda_{2}, \ldots, \lambda_{n}\right\}$. Then the normalized Laplacian spectrum of the double graph, $D_{2}(G)$, consists of 1 , repeats $n$ times and $1-\frac{\lambda_{i}}{r}$ for $i=1,2, \ldots, n$.

Proof. Let $A$ be the adjacency matrix of $G$. The Laplacian and normalized Laplacian matrix of $D_{2}(G)$ are

$$
L=\left[\begin{array}{cc}
2 r I_{n}-A & -A \\
-A & 2 r I_{n}-A
\end{array}\right] \text { and } \tilde{L}=\left[\begin{array}{cc}
I_{n}-\frac{A}{2 r} & \frac{-A}{2 r} \\
\frac{-A}{2 r} & I_{n}-\frac{A}{2 r}
\end{array}\right]
$$


As like the proof of the Theorem 3.2 and using Remark (2.1), we get the normalized Laplacian eigenvalues of $D_{2}(G)$.

\section{Applications}

In this section we discuss some applications of normalized Laplacian spectrum. Here we determine the normalized Laplacian energy, Kemeny's constant and number of spanning tree of the different graphs under consideration.

4.1. Normalized Laplacian energy. In [10], I. Gutman defined the graph energy, $E(G)$, as the sum of the absolute value of its eigenvalues. Let $G$ be a graph on $n$ vertices with adjacency spectrum $\lambda_{1} \geq \lambda_{2} \geq \cdots \geq \lambda_{n}$ then energy

$$
E(G)=\sum_{i=1}^{n}\left|\lambda_{i}\right|
$$

Let $G$ be a graph on $n$ vertices and normalized Laplacian spectrum $0=\sigma_{1} \leq \sigma_{2} \leq$ $\cdots \leq \sigma_{n}$. The normalized Laplacian energy is denoted by $\tilde{L} E(G)$ and is defined in [3] as

$$
\tilde{L} E(G)=\sum_{i=1}^{n}\left|\sigma_{i}-1\right| .
$$

Theorem 4.1. Let $G_{1}$ be an $r_{1}$ regular graph on $n_{1}$ vertices and $G_{2} \cong \bar{K}_{n_{2}}$, totally disconnected graph. Then,

$$
\tilde{L} E\left(G_{1} \nabla G_{2}\right)=2+\frac{2\left(E\left(G_{1}\right)-r_{1}\right)}{\sqrt{r_{1}\left(n_{2}+r_{1}\right)}} .
$$

Proof. We have $\lambda_{1}=r_{1}$ and $E(G)=\sum_{i=1}^{n_{1}}\left|\lambda_{1 i}\right|=r_{1}+\sum_{i=2}^{n_{1}}\left|\lambda_{1 i}\right|$. By Corollary 3.1 and (4.1) we get,

$$
\begin{aligned}
\tilde{L} E\left(G_{1} \bar{\nabla} G_{2}\right) & =n_{2} \times 0+2+\sum_{i=2}^{n_{1}} \frac{\left|\lambda_{1 i}\right|}{\sqrt{r_{1}\left(n_{2}+r_{1}\right)}}+\sum_{i=2}^{n_{1}} \frac{\left|-\lambda_{1 i}\right|}{\sqrt{r_{1}\left(n_{2}+r_{1}\right)}} \\
& =2+\frac{2}{\sqrt{r_{1}\left(n_{2}+r_{1}\right)}} \sum_{i=2}^{n_{1}}\left|\lambda_{1 i}\right| \\
& =2+\frac{2\left(E\left(G_{1}\right)-r_{1}\right)}{\sqrt{r_{1}\left(n_{2}+r_{1}\right)}} .
\end{aligned}
$$

Theorem 4.2. Let $G$ be a r-regular graph with $n$ vertices. Then

(a) $\tilde{L} E(\mathcal{D G})=\frac{2}{r} E(G)$;

(b) $\tilde{L} E\left(D_{2} G\right)=\frac{1}{r} E(G)$;

(c) $\tilde{L} E(\operatorname{splt}(G))=\frac{3}{2 r} E(G)$.

Proof. The proof follows from Theorem 3.2, Theorem 3.4 and Theorem 3.3. 
4.2. Kemeny's constant. Kemeny's constant $K(G)$, of a graph $G$ is defined as the expected number of steps required for the transition from a starting vertex $v_{i}$ called origin to a destination vertex, which is chosen randomly according to a stationary distribution of unbiased random walks on $G[2,7]$. Also $K(G)$ is a constant and is independent of the choice of the origin $v_{i}$. Let $G$ be a graph on $n$ vertices and normalized Laplacian spectrum $0=\sigma_{1} \leq \sigma_{2} \leq \cdots \leq \sigma_{n}$ then Kemeny's constant is the sum of all reciprocal normalized Laplacian eigenvalues except $1 / \sigma_{1}$. Thus we can write,

$$
K(G)=\sum_{i=2}^{n} \frac{1}{\sigma_{i}}
$$

Theorem 4.3. For $i=1,2$, let $G_{i}$ be $r_{i}$-regular graph on $n_{i}$ vertices with adjacency spectrum $\left\{r_{i}=\lambda_{i 1}, \lambda_{i 2}, \ldots, \lambda_{i n_{i}}\right\}$. Then the Kemeny's constant of $G_{1} \bar{\nabla} G_{2}$ is

$$
K\left(G_{1} \bar{\nabla} G_{2}\right)=\frac{\left(3 n_{1}+2 r_{2}\right)\left(n_{2}+r_{1}\right)}{2 n_{1} n_{2}+2 n_{1} r_{1}+n_{2} r_{2}}+\sum_{i=2}^{n_{2}} \frac{n_{1}+r_{2}}{n_{1}+r_{2}-\lambda_{2 i}}+\sum_{j=2}^{n_{1}} \frac{2 r_{1}\left(n_{2}+r_{1}\right)}{n_{2} r_{1}+r_{1}^{2}-\lambda_{1 j}^{2}} .
$$

Proof. Since for $i=1,2, G_{i}$ is $r_{i}$-regular graph on $n_{i}$ vertices and let $\eta_{1}$ and $\eta_{2}$ be the roots of the quadratic equation $x^{2}-\frac{3 n_{1}+2 r_{2}}{n_{1}+r_{2}} x+\frac{2 n_{1} n_{2}+2 n_{1} r_{1}+n_{2} r_{2}}{\left(n_{1}+r_{2}\right)\left(n_{2}+r_{1}\right)}=0$. Then

$$
\begin{aligned}
\frac{1}{\eta_{1}}+\frac{1}{\eta_{2}} & =\frac{\eta_{1}+\eta_{2}}{\eta_{1} \eta_{2}} \\
& =\frac{\left(3 n_{1}+2 r_{2}\right)\left(n_{2}+r_{1}\right)}{2 n_{1} n_{2}+2 n_{1} r_{1}+n_{2} r_{2}}, \\
K\left(G_{1} \bar{\nabla} G_{2}\right) & =\sum_{i=2}^{n_{2}} \frac{n_{1}+r_{2}}{n_{1}+r_{2}-\lambda_{2 i}}+\frac{1}{\eta_{1}}+\frac{1}{\eta_{2}} \\
& +\sum_{j=2}^{n_{1}}\left[\frac{\sqrt{r_{1}\left(n_{2}+r_{1}\right)}}{\sqrt{r_{1}\left(n_{2}+r_{1}\right)}+\lambda_{1 j}}+\frac{\sqrt{r_{1}\left(n_{2}+r_{1}\right)}}{\sqrt{r_{1}\left(n_{2}+r_{1}\right)}-\lambda_{1 j}}\right] \\
& =\frac{\left(3 n_{1}+2 r_{2}\right)\left(n_{2}+r_{1}\right)}{2 n_{1} n_{2}+2 n_{1} r_{1}+n_{2} r_{2}}+\sum_{i=2}^{n_{2}} \frac{n_{1}+r_{2}}{n_{1}+r_{2}-\lambda_{2 i}} \\
& +\sqrt{r_{1}\left(n_{2}+r_{1}\right)} \sum_{j=2}^{n_{1}} \frac{2 \sqrt{r_{1}\left(n_{2}+r_{1}\right)}}{r_{1}\left(n_{2}+r_{1}\right)-\lambda_{1 j}^{2}} .
\end{aligned}
$$

On simplification we get the required result.

Theorem 4.4. Let $G$ be an $r$-regular graph on $n$ vertices with adjacency spectrum $\left\{r=\lambda_{1}, \lambda_{2}, \ldots, \lambda_{n}\right\}$. Let $K(G)$ be the Kemeny's constant of $G$, then

(1) $K(\mathcal{D G})=K(G)+r \sum_{i=1}^{n} \frac{1}{r+\lambda_{i}}$;

(2) $K(\operatorname{splt}(G))=K(G)+2 r \sum_{i=1}^{n} \frac{1}{2 r+\lambda_{i}}$;

(3) $K\left(D_{2}(G)\right)=K(G)+n$. 
Proof. (1) Since $G$ is $r$-regular with adjacency spectrum $\left\{r=\lambda_{1}, \lambda_{2}, \ldots, \lambda_{n}\right\}$, the normalized Laplacian spectrum consists of $1-\frac{\lambda_{i}}{r}$, for $i=1,2, \ldots, n$. Therefore, $K(G)=\sum_{i=2}^{n}\left(1-\frac{\lambda_{i}}{r}\right)^{-1}$.

By Theorem 3.2 and (4.2) we get the Kemney's constant as

$$
\begin{aligned}
K(\mathcal{D G}) & =\sum_{i=2}^{n}\left(1-\frac{\lambda_{i}}{r}\right)^{-1}+\sum_{i=1}^{n}\left(1+\frac{\lambda_{i}}{r}\right)^{-1} \\
& =K(G)+r \sum_{i=1}^{n} \frac{1}{r+\lambda_{i}} .
\end{aligned}
$$

The other results obtained from Theorem 3.4, Theorem 3.3 and (4.2).

4.3. Number of spanning tree. Let $t(G)$ denote the number of spanning tree of the graph $G$, the total number of distinct spanning subgraphs of $G$ that are trees. If $G$ is a connected graph with $n$ vertices and the normalized Laplacian spectrum $0=\sigma_{1}(G) \leq \sigma_{2}(G) \cdots \leq \sigma_{n}(G)$ then the number of spanning tree (see [5])

$$
t(G)=\frac{\prod_{i=1}^{n} d_{i} \prod_{i=2}^{n} \sigma_{i}}{\sum_{i=1}^{n} d_{i}} .
$$

Theorem 4.5. For $i=1,2$ let $G_{i}$ be $r_{i}$-regular graph on $n_{i}$ vertices with adjacency spectrum $\left\{r_{i}=\lambda_{i 1}, \lambda_{i 2}, \ldots, \lambda_{i n_{i}}\right\}$. Then the number of spanning tree of $G_{1} \bar{\nabla} G_{2}$ is

$$
t\left(G_{1} \bar{\nabla} G_{2}\right)=r_{1} \prod_{i=2}^{n_{2}}\left(n_{1}+r_{2}-\lambda_{2 i}\right) \prod_{i=2}^{n_{1}}\left(n_{2} r_{1}+r_{1}^{2}-\lambda_{1 i}^{2}\right)
$$

Proof. Since for $i=1,2, G_{i}$ is a $r_{i}$-regular graph with $n_{i}$ vertices, there are $n_{1}$ vertices of degree $n_{2}+r_{1}$, another $n_{1}$ vertices are of degree $r_{1}$ and $n_{2}$ vertices are of degree $n_{1}+r_{2}$.

Let $\eta_{1}$ and $\eta_{2}$ be the roots of the quadratic equation

then we have

$$
x^{2}-\frac{3 n_{1}+2 r_{2}}{n_{1}+r_{2}} x+\frac{2 n_{1} n_{2}+2 n_{1} r_{1}+n_{2} r_{2}}{\left(n_{1}+r_{2}\right)\left(n_{2}+r_{1}\right)}=0,
$$

$$
\begin{aligned}
\eta_{1} \eta_{2} & =\frac{2 n_{1} n_{2}+2 n_{1} r_{1}+n_{2} r_{2}}{\left(n_{1}+r_{2}\right)\left(n_{2}+r_{1}\right)}, \\
\sum d_{i} & =n_{1}\left(n_{2}+r_{1}\right)+n_{1} r_{1}+n_{2}\left(n_{1}+r_{2}\right) \\
& =2 n_{1} n_{2}+2 n_{1} r_{1}+n_{2} r_{2}, \\
\prod d_{i} & =\left(n_{2}+r_{1}\right)^{n_{1}} r_{1}^{n_{1}}\left(n_{1}+r_{2}\right)^{n_{2}} .
\end{aligned}
$$

Hence, from (4.3), we get,

$$
\begin{aligned}
t\left(G_{1} \bar{\nabla} G_{2}\right) & =\frac{\left(n_{2}+r_{1}\right)^{n_{1}} r_{1}^{n_{1}}\left(n_{1}+r_{2}\right)^{n_{2}}}{2 n_{1} n_{2}+2 n_{1} r_{1}+n_{2} r_{2}} \eta_{1} \eta_{2} \prod_{i=2}^{n_{2}} \frac{n_{1}+r_{2}-\lambda_{2 i}}{n_{1}+r_{2}} \prod_{j=2}^{n_{1}} \frac{r_{1}\left(n_{2}+r_{1}\right)-\lambda_{1 j}^{2}}{r_{1}\left(n_{2}+r_{1}\right)} \\
& =r_{1} \prod_{i=2}^{n_{2}}\left(n_{1}+r_{2}-\lambda_{2 i}\right) \prod_{i=2}^{n_{1}}\left(n_{2} r_{1}+r_{1}^{2}-\lambda_{1 i}^{2}\right) .
\end{aligned}
$$


Theorem 4.6. Let $G$ be a r-regular graph on $n$ vertices with adjacency spectrum $\left\{r=\lambda_{1}, \lambda_{2}, \ldots, \lambda_{n}\right\}$. Let $t(G)$ be the number of spanning tree of $G$ then,

(1) $t(\mathcal{D G})=\frac{t(G)}{2} \prod_{i=1}^{n}\left(r+\lambda_{i}\right)$;

(2) $t(\operatorname{splt}(G))=\frac{t(G)}{3} \prod_{i=1}^{n}\left(2 r+\lambda_{i}\right)$;

(3) $t\left(D_{2}(G)\right)=2^{2 n-2} r^{n} t(G)$.

Proof. (1) Since $G$ is $r$-regular with adjacency spectrum $\left\{r=\lambda_{1}, \lambda_{2}, \ldots, \lambda_{n}\right\}$, the normalized Laplacian spectrum of $t(\mathcal{D G})$ consists of $1-\frac{\lambda_{i}}{r}$, for $i=1,2, \ldots, n$. Therefore $t(G)=\frac{1}{n} \prod_{i=2}^{n}\left(r-\lambda_{i}\right)$. Also $\prod_{i=2}^{n} d_{i}=r^{2 n}$ and $\sum_{i=1}^{n} d_{i}=2 n r$.

By Theorem 3.2 and (4.3) we get the

$$
\begin{aligned}
t(\mathcal{D G}) & =\frac{r^{2 n} \prod_{i=2}^{n} \frac{r-\lambda_{i}}{r} \prod_{i=1}^{n} \frac{r+\lambda_{i}}{r}}{2 n r} \\
& =\frac{t(G)}{2} \prod_{i=1}^{n}\left(r+\lambda_{i}\right) .
\end{aligned}
$$

The other results follows from Theorem 3.4, Theorem 3.3 and (4.3).

Acknowledgements. The author thankful to the University Grants Commission of Government of India for providing fellowship under the FDP in the XII plan.

\section{REFERENCES}

[1] A. Banerjee and J. Jost, On the spectrum of the normalzied graph Laplacian, Linear Algebra Appl. 428 (2008), 3015-3022.

[2] S. Butler, Algebraic aspects of the normalized Laplacian, in: A. Beveridge, J. Griggs, L. Hogben, G. Musiker and P. Tetali (Eds.), Recent Trends in Combinatorics, The IMA Volumes in Mathematics and its Applications, Springer International Publishing, Switzerland, Basel, 2016.

[3] M. Cavers, S. Fallat and S. Kirkland, On the normalized Laplacian energy and general Randic index $R_{-1}$ of graphs, Linear Algebra Appl. 433 (2010), 172-190.

[4] H. Chen and F. Zhang, Resistance distance and the normalized Laplacian spectrum, Discrete Appl. Math. 155 (2007), 654-661.

[5] F. R. K. Chung, Spectral Graph Theory, CBMS Regional Conference Series in Mathematics 92 and AMS, Providence, RI, 1997.

[6] D. M. Cvetković, M. Doob and H. Sachs, Spectra of Graphs, Theory and Applications, Third edition, Johann Ambrosius Barth, Heidelberg, 1995.

[7] J. J. Hunter, The role of Kemeny's constant in properties of Markov chains, Comm. Statist. Theory Methods 43 (2014), 1309-1321.

[8] G. Indulal and A. Vijayakumar, On a pair of equienergetic graphs, MATCH Commun. Math. Comput. Chem. 55 (2006), 83-90.

[9] S. Kirkland, Limit points for the normalzed Laplacian eigenvalues, Electron. J. Linear Algebra 15 (2006), 337-344.

[10] X. L. Li, Y. T. Shi and I. Gutman, Graph Energy, Springer-Verlag, New York, 2012.

[11] E. Sampathkumar, On Duplicate Graphs, J. Indian Math. Soc. 37 (1973), 285-293.

[12] E. Sampathkumar and H.B. Walikar, On Splitting Graph of a Graph, Journal of Karnatak University (Science) 25(13) (1980), 13-16.

[13] R. P. Varghese and D. Susha, Spectrum of some new product of graphs and its applications, Global Journal of Pure and Applied Mathematics 13(9) (2017), 4493-4504. 
[14] R. P. Varghese and D. Susha, The spectrum of two new corona of graphs and its applications, International Journal of Mathematics and its Applications 5(4) (2017), 395-406.

${ }^{1}$ Department of Mathematics,

Catholicate College, Pathanamthitta

Kerala, India - 689645

Email address: rennypv1@gmail.com

Email address: sushad70@gmail.com 